
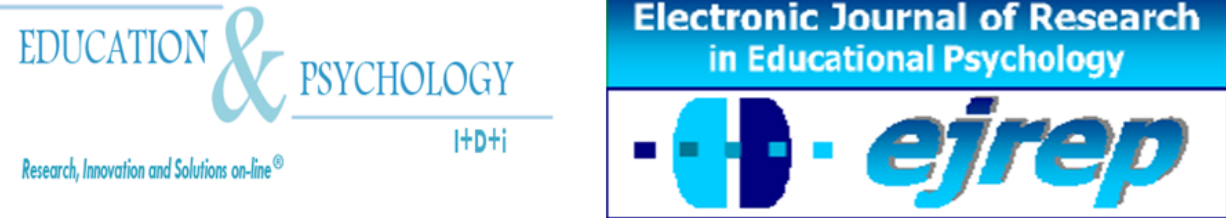

Editorial EOS

\title{
Evaluación del laboratorio de orientación educativa como escenario de práctica en un modelo curricular basado en competencias
}

\section{Jesús Juan Francisco Arredondo Damián, M.C.E. Claudia Cecilia Norzagaray Benítez}

Departamento de Psicología y CC. de la Comunicación, Universidad de Sonora, Hermosillo

\section{México}

Correspondencia: Jesús Juan Francisco Arredondo Damián. Angel García Aburto \#390. Entre Olivares y Loaiza. Colonia Olivares. C.P. 83180- Hermosillo, Sonora. México. E-mail: giovanni_1986@hotmail.com

(C) Education \& Psychology $\mathrm{I}+\mathrm{D}+\mathrm{i}$ and Editorial EOS (Spain) 


\section{Resumen}

Introducción. El objetivo principal de éste estudio es describir la percepción de los participantes, respecto al uso del Laboratorio de Orientación Educativa como escenario de prácticas de alumnos de tercer semestre de la psicología de la Universidad de Sonora (México) quienes deben mostrar las competencias de aplicación de instrumentos psicométricos del ámbito de educación.

Método. La muestra está compuesta por tres grupos: 189 estudiantes de tercer semestre de la Licenciatura en Psicología de la Universidad de Sonora, 5 profesores que impartieron la materia Práctica Supervisada I y 15 supervisores del Laboratorio de Orientación Educativa

Resultados. Los resultados destacan que los estudiantes, profesores y supervisores del escenario, consideran que se cumplió con el objetivo de aplicar el instrumento s2000 plus. En cuanto al manual de entrenamiento, todos los involucrados consideran que fue una herramienta útil para el aprendizaje del instrumento. Los resultados mas contrastantes son las calificaciones acerca del espacio físico del LOE. Los supervisores son los que se muestran más inconformes ya que $60 \%$ seleccionaron las calificaciones mas bajas, 1 y 2 . Acerca de los estudiantes, el $70.2 \%$ de ellos califica con 4 o 5 el espacio del LOE.

Conclusiones. Se concluye que el Laboratorio de Orientación Educativa está cumpliendo con sus funciones para desarrollar la competencia de aplicación de instrumentos psicométricos resultando una experiencia útil y educativa para los alumnos como su primera práctica con usuarios de servicios psicológicos. Así mismo, el manual de entrenamiento diseñado para dichos fines ha sido valorado como útil y facilitador de la transición de la práctica en aula a la aplicación con los usuarios.

Palabras Clave: aplicación de instrumentos, plan de estudios, competencias, escenarios de práctica, práctica supervisada.

Recibido: 03/01/09 Aceptación inicial: 08/01/09 Aceptación final: 27/04/09 


\title{
Assessment of an Educational Guidance Laboratory as a prac- tical setting within a curriculum model based on competencies
}

\begin{abstract}
Introduction. The main objective of this study is to describe the perception of participants in respect to the use of the Laboratorio de Orientación Educativa as scenario practice of students of third semester of psychology from the Universidad de Sonora (México) who must demonstrate the skills of application of psychometric instruments in the field of education..
\end{abstract}

Method. The sample consists of three groups: 189 students of third semester of the Licence in Psychology at the University of Sonora, 5 teachers who taught the subject Supervised Practice I and 15 supervisors Laboratorio de Orientación Educativa

Results. The results show that students, teachers and supervisors of the scenario, considered to be fulfilled in order to implement the instrument s2000 plus. Regarding the training manual, all involved believe that was a useful tool for learning. The most contrasting results are the ratings about the fisical space of the LOE. Supervisors are the most dissatisfied and $60 \%$ selected the lower grades, 1 and 2 . About the students, the $70.2 \%$ of them qualify with 4 or 5 LOE space

Conclusions. We conclude that the Laboratorio de Orientación Educativa is fulfilling its responsibilities to develop the competence of implementation of psychometric instruments and a useful educational experience for students as their first practice with users of psychological services. Likewise, the training manual designed for those purposes has been rated as useful and facilitates the transition from classroom to practice in the application with users.

Keywords: application of tools, curriculum, skills, practice scenarios, supervised practice.

Received: 01/03/09 Initial Acceptance: 01/08/09 Definitive Acceptance: 04/27/09 


\section{Introducción}

El proyecto de Macotela y Paredes (2003) de un modelo para la intervención psicológica en escenarios educativos establece claramente la necesidad de que los futuros profesionistas sean formados en escenarios reales y supervisados por expertos en la temática propia del escenario así como que dicha supervisión debe gradualmente desaparecer.

Uno de los supuestos de Macotela (2007) es que además de insertarse fluidamente en el mercado laboral, los estudiantes o profesionales de la psicología deben involucrarse en escenarios con proyectos formales que desarrolle habilidades profesionales y realización de investigación en dichos escenarios, apoya la difusión del campo de acción del psicólogo y a generar una mayor necesidad de su participación. Es claro que el conocimiento teórico es indispensable, pero es mas claro aún que carece de significado cuando no es vinculado rotundamente con problemáticas reales y socialmente relevantes.

En las modalidades de los nuevos planes de estudio que giran alrededor del desarrollo de competencias se hace hincapié en la importancia de aproximar lo más posible a los estudiantes a las problemáticas sociales reales, para que las competencias desarrolladas sean acordes a éste sentido.

El Plan de Estudios actual de la Licenciatura en Psicología (PEP) (2004) esta basado en modelos de formación por competencias y está organizado en cinco ejes formativos: Común, Básico, Profesional, Especializante e Integrador, de acuerdo con los ejes curriculares adoptados por la Universidad de Sonora.

Entre los lineamientos didácticos propuestos por el plan de estudios destacan para el presente escrito los siguientes: Se plantean dos situaciones básicas para la formación profesional: prácticas y seminarios.

Las prácticas son situaciones en las que el estudiante debe desempeñarse ante los objetos o situaciones referentes del discurso didáctico haciendo, en forma individual o grupal, lo necesario para cumplir el criterio de logro especificado; dichas prácticas se han planeado para que se transite de la práctica bajo situaciones controladas de laboratorio a la práctica en el campo profesional real. Por su naturaleza, las prácticas no admiten mucha diversidad en cuan- 
to a modalidades educativas se refiere, prefiriéndose la presencial en virtud de la necesidad del contacto directo con las situaciones problema y la supervisión y retroalimentación constante por parte del profesor.

Por su parte, los seminarios son situaciones que generalmente ocurren en el aula, donde el estudiante entra en contacto con información necesaria (discurso didáctico) para contextualizar su desempeño práctico y se socializa activamente dentro del grupo de trabajo. Los seminarios sí pueden admitir diferentes modalidades del "sistema educativo" — presencial, a distancia o distribuido - dependiendo de la disponibilidad de las fuentes de información y los medios de comunicación con los que se cuente (PEP, 2004).

Decir que un estudiante o un profesional de la psicología son competentes, para Piña y Rivera (2006) es equivalente a decir que disponen de los conocimientos teóricos y metodológicos disciplinarios, pertinentes a las circunstancias sociales en las que los conocimientos pueden ser potencialmente aplicables; competencia es un termino disposicional que habla de la probabilidad de.

El PEP 2004 pretende que el profesor mediante el ejemplo, discurso y práctica supervisada en situaciones reales de aplicación y ejercicio de lo que enseña, genere en el estudiante habilidades y competencias en contextos y situaciones que impriman sentido disciplinario y social a su quehacer como aprendiz, para lo cual adoptó una estrategia basada en competencias para el nuevo plan de estudios. Dicho plan se basó en el modelo de interacciones didácticas de Ibáñez (2004), el cual plantea el diseño curricular en base a competencias conductuales fundamentándose en la psicología interconductual y empleando la taxonomía de Ribes y López (1985) para describir las situaciones idóneas para el establecimiento de competencias.

El modelo de interacciones didácticas fue creado como una propuesta para superar las dificultades teóricas o errores categoriales que adolece el modelo del triángulo pedagógico o interactivo. Entre las principales críticas al modelo del triangulo didáctico se encuentra la concepción de que el conocimiento es una "cosa" y que, como consecuencia esa "cosa" puede ser adquirida (aprender), lo cual genera una adquisición de conocimiento con la mediación, transmisión o facilitación del profesor (Ibáñez, 2007a). 
Las interacciones didácticas son las relaciones que se establecen entre los agentes y factores de los procesos educativos durante un tiempo y lugar determinado y organizado expresamente para proveer y generar condiciones propicias que permitan el aprendizaje del estudiante (episodio instruccional).

Así mismo Ibáñez (2007b) refiere el término de competencias como una disposición a hacer y decir ante las situaciones concretas del mundo real de acuerdo con los criterios convencionales de una determinada comunidad epistémica (o desempeño efectivo). Además, adentrándose en el contexto educativo formal dice que una competencia es el desempeño mostrado por un alumno ante una determinada tarea y que, regularmente, es efectivo en lograr el criterio requerido.

Las competencias son establecidas como capacidades por Ribes citado en Ibáñez (2007a), es decir, a acciones que producen resultados o logros específicos en situaciones en las que se requiere de alguna destreza o habilidad específica para alcanzar dichos logros. Para concebir la naturaleza de una competencia existen tres dimensiones: dominio, intensidad del dominio competencial y el nivel de aptitud funcional. Dominio tiene que ver con la morfología empleada según la tarea a resolver mediante los conocimientos de una determinada disciplina. La intensidad del dominio competencial se refiere al dominio que se tiene de una competencia respecto a aspectos cuantitativos como el nivel de especialización necesario para resolver una problemática. El nivel de aptitud funcional es reconocido por la manera en que se configura el desempeño ante una determinada tarea así como por el grado de dependencia del comportamiento respecto a la situación en la que se realiza una tarea. Ribes y López (1985) identifican cinco diferentes niveles de organización funcional en los que puede desarrollarse una competencia. (Ibáñez, 2007a).

En concordancia con la taxonomía propuesta por Ribes y López (1985) una misma competencia se entiende que puede ser desarrollada en uno o más de los cinco niveles de organización funcional. Si se pretende que el alumno no se limite a referir, narrar o describir sino que sean competentes en razonamientos científicos, juicios y análisis, se debe de desarrollar dicha competencia en los 5 niveles funcionales de menor a mayor complejidad llegando hasta el nivel sustitutivo no referencial (Guevara, 2006). La competencia a desarrollar en la materia Práctica Supervisada I es la de aplicación, dicha competencia corresponde al nivel suplementario planteado en la taxonomía de Ribes y López (1985). Para el aprendizaje de 
competencias en el nivel suplementario es fundamental la ocurrencia de las acciones siendo reguladas estrictamente por el discurso, en presencia de las condiciones ambientales necesarias para que éstas puedan realizarse (Ibáñez, 2007a). Lo cual es exactamente lo que se realiza en la Práctica Supervisada I.

Una competencia no es lograda en un alumno enseñándole el que, sino el como (Guevara, 2006). La práctica por repetición o hábito y la enseñanza por imitación no fomentan el desarrollo del comportamiento inteligente. Para generar condiciones idóneas para el desarrollo de competencias profesionales (capacidad de hacer), conceptuales o discursivas (capacidad de decir) e instrumentales (capacidad de usar), las cuales componen el desempeño disciplinar, Ibáñez (2007a) plantea tres tipos de asignaturas que deben existir dentro de un plan de estudios: en las prácticas se plantean situaciones problema para que sean resueltas en base a sus conocimientos por el estudiante, los seminarios constituyen los contenido referenciales del discurso didáctico y los talleres permiten al estudiante hacer contacto con propiedades particulares del objeto de estudio mediante herramientas, instrumentos o aparatos a emplear durante el desempeño competencial. El Plan de Estudios de Psicología 2004 solo plantea las prácticas y los seminarios, mas sin embargo, todas las características de las que constan los talleres se encuentran incluidas en los seminarios y prácticas.

En la actualidad, de acuerdo con Macotela (2007) para lograr una formación adecuada de profesionales en cuanto a que cuenten con las competencias propias de su profesión, es necesario trabajar directamente en escenarios reales, con problemáticas acordes al entorno social.

A partir de la entrada del PEP-2004 hay un cambio de plan curricular el cual, para ejecutarse de manera adecuada demanda varios escenarios para el establecimiento de competencias en el estudiante. Particularmente en la materia de Practica Supervisada I, la competencia a desarrollar es la aplicación de instrumentos ya sea de manera presencial o sucedánea.

Las prácticas que se realizan en cada semestre del PEP 2004 son situaciones en las que el estudiante debe desempeñarse ante los objetos o situaciones referentes del discurso didáctico haciendo, en forma individual o grupal, lo necesario para cumplir el criterio de logro especificado; dichas prácticas se han planeado para que se transite de la práctica bajo situaciones controladas de laboratorio a la práctica en el campo profesional real. 
Macotela (2007) refiere que es inadecuado suponer que es suficiente darle indicaciones a un estudiante sobre casos hipotéticos para que actúe de determinada manera cuando se requiera. La estrategia por competencias adoptada por el PEP-2004 implica la búsqueda de escenarios pertinentes, entre los cuales debido a su disposición se encuentran dentro de la misma universidad como el Laboratorio Organizacional (LABORG) y el Laboratorio de Orientación Educativa (LOE).

El Programa de Práctica Supervisada I (2007) plantea que la materia es un espacio educativo correspondiente al tercer semestre cuyo objetivo general es que el alumno sea capaz de obtener información sobre las interacciones psicológicas empleando con efectividad instrumentos, técnicas y procedimientos de observación, registro y medición pertinentes a los distintos niveles funcionales en situaciones sucedáneas y naturales en las modalidades: Individuo-con otros Individuos, Individuo-Medio físico, Individuo-consigo mismo e Individuoinstituciones, en cada ámbito de práctica profesional (salud, medio ambiente, convivencia social, educación y producción y consumo), mostrando objetividad, discreción, responsabilidad, confidencialidad y profesionalismo en el manejo de información.

Actualmente hay varios escenarios que figuran como responsables en la aplicación de instrumentos, entre los cuales figura el LOE, el cual se puede clasificar en base a Peralta (2005) como orientación de nivel de centro educativo y que tiene como objetivos específicos reforzar en materia de orientación vocacional a las Instituciones de Educación Media y Media Superior y a la misma Universidad de Sonora; tener acceso a la tecnología de vanguardia en materia de orientación vocacional y profesional; y, propiciar actividades prácticas en técnicas de evaluación del recurso humano conjugando los aspectos conceptuales, metodológicos y tecnológicos. Cada año en que se imparte la asignatura dichos escenarios tanto los internos como externos a la universidad cubren la función de recibir alumnos que aplicarán lo aprendido en aulas, exponiéndolas a una situación real del trabajo del psicólogo. En el ciclo 2007-2 el LOE diseñó un proyecto de servicio en el cual se evaluaron a los alumnos de primer semestre de la Licenciatura en Ciencias de la comunicación de la Universidad de Sonora con la finalidad de identificar el perfil vocacional de cada uno y analizar la correspondencia de este con el perfil ideal de ingreso, detectando áreas fuertes y de oportunidad. Esta información se envia a los tutores como apoyo a sus posibles actividades. Para elaborar el perfil se aplicó la técnica de la entevista y el sistema 2000 el cual consta de cuatro fases: sección computarizada, inventario, muestras de trabajo y un video; estas fases fueron en las que se capacitaron a los alum- 
nos en aulas para que lo transfirieran a la aplicación del mismo en una situación real. El instrumento, espacio, materiales y el manual de entrenamiento para el trabajo en aula fue diseñado por responsables de LOE.

De ahí que surja la relevancia de evaluar los escenarios que se usan como escenarios de prácticas para verificar que estén cumpliendo con su función. Por eso es importante conocer la percepción de los figurantes pertinentes, así como que los escenarios favorezcan el desarrollo de competencias. Sander (2005) refiere que no solo es importante saber acerca del conocimiento específico de las asignaturas por parte de los alumnos, sino tambien sus concepciones y percepciones en cuanto a la enseñanza y el aprendizaje.

Evaluaciones realizadas en planes de estudios basados en competencias muestran que en cuanto a competencias como la de identificación de conceptos no hay diferencias significativas en grupos de primero y tercer semestre con relación a la adquisición de dicha competencia, pese a que hay una diferencia de un año mas de entrenamiento escolar (Frias y Jasa, 2005).

Ésta investigación es de una utilidad práctica ya que por medio de la percepción que tienen los figurantes pertinentes en base a la relación que tuvieron con las actividades realizadas en el laboratorio y en las que participaron diversas figuras (estudiantes, maestros y supervisores de escenario), existe la posibilidad de realizar sugerencias propositivas con la finalidad de mejorar el uso del LOE como escenario de prácticas de estudiantes de la Licenciatura en Psicología. La continua necesidad de perfeccionar la implementación del PEP-2004 es una de los principales incitadores para investigar acerca de la utilización de los escenarios disponibles para la realización de las prácticas, sin asumir de antemano la relevencia y utilidad de las mismas.

Con el desarrollo de la competencia de aplicación de instrumentos y procedimientos psicológicos se posibilita que el alumno despliegue habilidades en la recolección de información necesarias para el diagnóstico de problemáticas dentro de la disciplina psicológica.

Debido a que al ser el primer contacto por parte de los estudiantes con usuarios de servicios psicológicos es de gran importancia describir y analizar la percepción de las figuras pertinentes en esa transición de simulacros y casos escritos a usuarios verdaderos. 
Los objetivos fundamentales del presente trabajo son (a) describir la percepción de los estudiantes, profesores que imparten la materia Práctica Supervisada I y supervisores del LOE en aspectos concernientes al uso del laboratorio como escenario de prácticas y si se cumplen con los objetivos de aplicación de instrumentos en el ámbito de educación, (b) analizar las concordancias y discordancias entre la percepción de los estudiantes, profesores que imparten la materia Práctica Supervisada I y supervisores del LOE en aspectos relacionados al uso del LOE como escenario de prácticas, (c) describir sugerencias derivadas de la percepción de los estudiantes, profesores que imparten la materia Práctica Supervisada I y supervisores del LOE en aspectos relacionados al uso del LOE como escenario de prácticas.

\section{Método}

\section{Diseño de investigación}

La presente es una investigación no experimental, transeccional descriptiva.

\section{Participantes}

Las poblaciones que se estudia se dividen en 3 grupos: 189 estudiantes de tercer semestre de la Licenciatura en Psicología de la Universidad de Sonora, 5 profesores que impartieron la materia Práctica Supervisada I y 15 supervisores del LOE. Los alumnos se encontraban en el ciclo escolar 2007-2.

Para ésta investigación en cuanto a la población de profesores que impartieron la materia Práctica Supervisada I se tomó una muestra de 5 profesores debido a su disponibilidad. En cuanto a las poblaciones de estudiantes de tercer semestre y supervisores del espacio, no se tomó una muestra, sino que se utilizaron las poblaciones completas.

\section{Instrumento}

El instrumento empleado es un formato de realimentación del escenario en tres versiones, adecuadas para estudiantes, maestros y supervisores. El formato para estudiantes consta de 15 reactivos de los cuales 3 de ellos son dicotómicos, 6 de ellos son preguntas abiertas y 6 de ellos son reactivos en escala Likert que van del 1 al 5 siendo 1 el mínimo y 5 el máximo posible. El formato para profesores consta de 9 reactivos de los cuales 3 de ellos son dicotó- 
micos y 6 son preguntas abiertas. El formato para supervisores consta de 12 reactivos de los cuales 2 de ellos son dicotómicos, 4 de ellos son preguntas abiertas y 6 de ellos son reactivos en escala Likert que van del 1 al 5 siendo 1 el mínimo y 5 el máximo posible.

En cuanto a los reactivos que se contestan de manera abierta, en base a las respuestas obtenidas se realizaron categorías para agrupar las respuestas para cada uno de esos reactivos. Dichas categorías las realizaron los supervisores del espacio.

Se evalúan temáticas relacionadas con el cumplimiento de objetivos, utilidad del manual de entrenamiento, calidad de servicio y atención tanto a los practicantes como usuarios. Se solicitan sugerencias de mejoras.

Respecto a la validez y fiabilidad del instrumento se sometió a la evaluación de un panel de expertos académicos de la universidad con amplia experiencia en el ámbito de la educación y orientación educativa, ello antes de la aplicación para que hicieran los aportes necesarios y se verificó si la construcción y el contenido del instrumento son adecuados para obtener datos de las variables a investigar. En cuanto al nivel de confiabiliad se empleó el alfa de Cronbach para el análisis, el cual arrojo un índice de .70 lo cual se considera aceptable.

\section{Procedimiento}

A manera de precedente, antes de recabar los datos, las prácticas en el LOE habían concluido exitosamente; los estudiantes demostraron ser competentes en la aplicación del Sistema 2000 Plus y ello fue certificado en primera instancia por los profesores del la materia Práctica Supervisada I en las aulas antes de acudir al escenario y en segunda instancia por los supervisores del escenario una vez que aplicaron el instrumento de manera exitosa.

La recopilación de los datos de los estudiantes se llevó a cabo en las aulas de clase en la materia Práctica Supervisada I y fue realizada por los mismos profesores usando el formato de realimentación del escenario para estudiantes. Los datos de los supervisores y profesores fueron recabados de manera presencial o por correo electrónico empleando el formato de realimentación del escenario correspondiente. 


\section{Análisis de datos}

El análisis de los datos recabados se realizó mediante el empleo del paquete estadístico Statistical Package for the Social Sciences (SPSS) versión 13 para Windows.

\section{Resultados}

\section{Estudiantes.}

En la información proporcionada por los estudiantes se encuentran considerables datos que no fueron tomados como perdidos sino agrupados en una categoría de respuestas no relacionadas con la pregunta o afectiva. El origen de dicha categoría responde al hecho de que muchas respuestas fueron simplemente la reformulación de la pregunta de manera afirmativa o expresiones de emociones que no corresponden a la pregunta en sí.

El 94.7\% de los estudiantes consideran que se logró el objetivo de aplicar el s2000 plus, solo el 5.3\% no lo considera así. En cuanto al “¿por qué?” de ello, el 21.9\% contestó una respuesta no relacionada con la pregunta o se dio una respuesta afectiva. Un $20.2 \%$ de los estudiantes contestó que aprendieron acerca de las áreas que evalúa el s2000 plus y 19.1\% que aprendieron a aplicar las evaluaciones. El resto de respuestas se agrupan en una categoría de varias opciones pues de manera individual no superaban el 15\% en cada categoría de respuesta, dichas respuestas fueron las siguientes: no hubo evaluados y las instrucciones se les dieron a los supervisores en un juego de roles, existe un ambiente relajado, obtuve todas las firmas del formato de guía de entrenamiento, por la elaboración de un reporte del perfil del evaluado, todas las prácticas se realizaron con evaluados reales, se realizó todo lo planeado en el escenario.

Con respecto a la correspondencia entre el material visto en la asignatura de Práctica Supervisada I con el trabajo realizado en el escenario, los estudiantes en un $98.9 \%$ asegura que si existe correspondencia, mientras que solo el $1.1 \%$ asegura lo contrario. Con respecto a la causa, el 62.8\% indica que el s2000 plus se aplicó en el escenario de manera muy similar al aula de clases, $19.7 \%$ de los estudiantes aseveran que las indicaciones que se llevaron a cabo en el espacio corresponden a las del manual de entrenamiento del s2000 plus; por el contrario $8.7 \%$ disienten asegurando que las indicaciones no corresponden completamente a dicho ma- 
nual. Habiendo seleccionado a cada una menos del 5\% de los estudiantes se encuentran las respuestas no relacionadas a la pregunta o afectivas.

La totalidad de los estudiantes reportan que el manual de entrenamiento del s2000 plus fue una herramienta útil para su aprendizaje. Las principales razones, aseguran que es debido a que cuentan con información buena, útil y detallada (51.6\%) y que con apoyo del manual se facilitó la práctica y el aprendizaje (35.3\%). El 10\% de los estudiantes encuentran que algunas instrucciones varían con respecto a las del manual y respuestas no relacionadas a la pregunta o afectivas.

Entre los aspectos interesantes o positivos del espacio destacados por los estudiantes se encuentran los instrumentos (21.5\%), las instalaciones (14.5\%), los supervisores $(14.5 \%)$, cargado de datos $(8.1 \%)$, procedimientos $(7.6 \%)$ y contacto con los usuarios $(7 \%)$. En cuanto a las respuestas no relacionadas con la pregunta o respuestas afectivas el porcentaje fue de $16.3 \%$. Con menos de 5\% de los estudiantes habiendo seleccionado cada una se encuentran las siguientes categorías de respuesta: organización, atención y resultados.

Los estudiantes también hicieron sugerencias generales para mejorías del espacio como escenario de prácticas y las mas frecuentes fueron en relación a que se necesitan más áreas dónde evaluar (34.1\%), un mayor respeto por parte de los supervisores $(8.5 \%)$ y más equipo (7.3\%). También, con menos del 5\% de estudiantes que las seleccionaron por cada una se encuentran las categorías de mayor puntualidad, mas cantidad de ensayos en el aula antes de ir al espacio, menos ruido y que se pongan de acuerdo los profesores de la materia y los supervisores del espacio. El $25.6 \%$ de los estudiantes emplearon una respuesta no relacionada con la pregunta o de índole afectiva

La experiencia dentro del escenario fue valorada por los estudiantes en general buena o positiva (68.1\%) además de útil y enriquecedora (14.1\%). Con menos del 10\% de estudiantes se encuentran las categorías de respuesta de educativa y negativa; el 14.1\% contestaron de una manera que no se relacionaba su respuesta a la pregunta o de manera afectiva. 


\section{Supervisores.}

La totalidad de los supervisores del espacio consideran que se cumplió con el objetivo de que los estudiantes aplicaran el s2000 plus. Esto es atribuido a que realizaron todo lo planeado en el escenario (80\%) y en menor medida a que aprendieron a aplicar las evaluaciones (13.3\%) y que toda la práctica se llevó a cabo con evaluados reales $(6.7 \%)$.

Todos los supervisores concuerdan con que el manual de entrenamiento del s2000 plus es una herramienta útil para el aprendizaje de los estudiantes, el 46.7\% lo atribuye a que con su apoyo se facilitó la práctica y el aprendizaje, el 33.3\% a que cuenta con información buena, útil y detallada, $13.3 \%$ considera que algunas instrucciones varían con respecto al manual y solo el $6.7 \%$ lo atribuye a que es útil para el cargado de datos.

Las sugerencias generales para mejorías del espacio como escenario de prácticas fueron tres, la mas destacada fue la necesidad de mas espacio donde evaluar con un $86.7 \%$, seguida de que practiquen todo lo que se hace en el LOE y más recursos materiales para el espacio con $6.7 \%$ cada una.

\section{Profesores}

La totalidad de la muestra de los profesores considera que se cumplió con el objetivo de que los estudiantes aplicaran el s2000 plus debido a que aprendieron a aplicar las evaluaciones $(66.7 \%)$ y a que realizaron todo lo planeado en el escenario $(33.3 \%)$.

En cuanto a la correspondencia entre el material visto en la asignatura de Practica Supervisada I con el trabajo realizado en el escenario, toda la muestra concuerda con que existe dicha correspondencia, esto atribuyéndolo a las siguientes causas: Lo revisado en clase corresponde a lo hecho en el escenario (40\%), el material es de fácil aprendizaje, práctico y gran apoyo (20\%), se cumplió con el objetivo de aplicar el instrumento (20\%) y a que lo ejercitado en clases con el manual es de una gran utilidad práctica (20\%).

La muestra de profesores también coincidió en que el manual de entrenamiento del s2000 plus fue una herramienta útil para el aprendizaje de los estudiantes, esto según en $40 \%$ de ellos debido a que con su apoyo facilitó la práctica y el aprendizaje, otro 40\% lo atribuyó a 
que cuenta con buena información, muy útil y detallada. Finalmente $20 \%$ de la muestra asegura que es debido a su utilidad para el cargado de datos.

Cuando se les cuestionó acerca de los aspectos que les parecieron interesantes o positivos respecto al espacio, los resultados fueron: organización (60\%), instrumentos (30\%) y el ejemplo que es el trabajo realizado en el LOE para los estudiantes $(20 \%)$.

Las sugerencias generales por parte de los profesores para mejorías del espacio como escenario de prácticas fueron las siguientes: más áreas donde evaluar (20\%), que todas las secciones de la evaluación sean aplicadas por un practicante a un solo evaluado (20\%), más acuerdos entre los profesores de la materia y los responsables del espacio (20\%) y que los estudiantes practiquen todas las tareas que son realizadas en el LOE (20\%). La experiencia de los estudiantes dentro del espacio fue valorada por los profesores como útil y enriquecedora o de experiencia (60\%) y en general buena o positiva (40\%).

A los estudiantes y supervisores se les pidió que respondieran en una escala del 1 al 5, donde 5 es el máximo puntaje y 1 el mínimo en aspectos relacionados con la calidad del servicio prestado, específicamente en puntos acerca de puntualidad, atención y servicio, respeto a los evaluados, respeto a los practicantes, suficiencia de equipo y espacio con respecto al LOE. (Tabla 1)

Tanto los estudiantes como los supervisores del espacio en cuanto a los aspectos de puntualidad, atención y servicio, el respeto a los evaluados y respeto a los practicantes coinciden en puntajes elevados oscilando en el 4 y 5 , siendo generalmente los supervisores los que otorgan mejores calificaciones.

En cuanto a la suficiencia de equipo las calificaciones tanto de estudiantes como supervisores se encuentran en su mayoría entre el 3 y el 5 . Cabe destacar que $20 \%$ de los supervisores calificaron con 5 la suficiencia del equipo, en cambio más del doble (42.24\%) de los estudiantes otorgaron dicha calificación.

Los resultados mas contrastantes se encuentran en las calificaciones otorgadas al espacio físico del LOE. Los supervisores son los que se muestran más inconformes ya que $60 \%$ 
seleccionaron las calificaciones mas bajas, 1 y 2 . En cuanto a los estudiantes, el $70.2 \%$ de ellos califica con 4 o 5 el espacio del LOE

La experiencia dentro del escenario fue valorada en un $53.3 \%$ como buena o positiva, $33.3 \%$ la valoraron como útil y enriquecedora, el restante $13.3 \%$ la valoraron como educativa. Los datos de los tres grupos frecuentemente concuerdan entre sí aunque en diferente proporción, tanto de concordancias y discordancias como de las proporciones con que ocurren pueden hacerse una serie de hipótesis sobré las razones por las que percibieron de tal o cual manera ésa variable.

Tabla 1. Evaluación de la calidad de servicio, factores y grupo

\begin{tabular}{|c|c|c|c|}
\hline FACTOR & $\overline{\text { GRUPO }}$ & $\begin{array}{l}\text { PUNTAJE OBTENI- } \\
\text { DO AL CALIFICAR }\end{array}$ & $\%$ de Sujetos \\
\hline \multirow{10}{*}{ Puntualidad } & \multirow{5}{*}{ Estudiantes } & 1 & 5.88 \\
\hline & & 2 & 10.69 \\
\hline & & 3 & 10.69 \\
\hline & & 4 & 35.82 \\
\hline & & 5 & 36.89 \\
\hline & \multirow{5}{*}{ Supervisores } & 1 & 0 \\
\hline & & 2 & 0 \\
\hline & & 3 & 13.33 \\
\hline & & 4 & 53.33 \\
\hline & & 5 & 33.33 \\
\hline \multirow{10}{*}{$\begin{array}{l}\text { Atención } \quad y \\
\text { Servicio }\end{array}$} & \multirow{5}{*}{ Estudiantes } & 1 & 3.19 \\
\hline & & 2 & 7.97 \\
\hline & & 3 & 14.36 \\
\hline & & 4 & 29.25 \\
\hline & & 5 & 45.21 \\
\hline & \multirow{5}{*}{ Supervisores } & 1 & 0 \\
\hline & & 2 & 0 \\
\hline & & 3 & 0 \\
\hline & & 4 & 26.66 \\
\hline & & 5 & 73.33 \\
\hline \multirow{10}{*}{$\begin{array}{l}\text { Respeto a los } \\
\text { Evaluados }\end{array}$} & \multirow{5}{*}{ Estudiantes } & 1 & 1.62 \\
\hline & & 2 & 3.24 \\
\hline & & 3 & 4.86 \\
\hline & & 4 & 20 \\
\hline & & 5 & 70.27 \\
\hline & \multirow{5}{*}{ Supervisores } & 1 & 0 \\
\hline & & 2 & 0 \\
\hline & & 3 & 0 \\
\hline & & 4 & 13.33 \\
\hline & & 5 & 86.66 \\
\hline
\end{tabular}




\begin{tabular}{|c|c|c|c|}
\hline & & & \\
\hline & & 1 & 3.24 \\
\hline & & 2 & 6.48 \\
\hline & Estudiantes & 3 & 15.13 \\
\hline & & 4 & 21.08 \\
\hline Respeto a los & & 5 & 54.05 \\
\hline Practicantes & & 1 & 6.66 \\
\hline & & 2 & 6.66 \\
\hline & Supervisores & 3 & 6.66 \\
\hline & & 4 & 26.66 \\
\hline & & 5 & 60 \\
\hline Suficiencia de & & 1 & 1.06 \\
\hline Equipo & & 2 & 3.2 \\
\hline & Estudiantes & 3 & 21.92 \\
\hline & & 4 & 31.55 \\
\hline & & 5 & 42.24 \\
\hline & & 1 & 0 \\
\hline & & 2 & 0 \\
\hline & Supervisores & 3 & 33.33 \\
\hline & & 4 & 46.66 \\
\hline & & 5 & 20 \\
\hline Espacio & & 1 & 5.85 \\
\hline & & 2 & 10.63 \\
\hline & Estudiantes & 3 & 13.29 \\
\hline & & 4 & 32.44 \\
\hline & & 5 & 37.76 \\
\hline & & 1 & 26.66 \\
\hline & & 2 & 33.33 \\
\hline & Supervisores & 3 & 20 \\
\hline & & 4 & 13.33 \\
\hline & & 5 & 6.66 \\
\hline
\end{tabular}

\section{Discusión y Conclusiones}

De los resultados y la comparación de ellos en base a los grupos se puede concluir que el LOE cumple adecuadamente como un escenario de práctica además de lo siguiente:

Tanto supervisores como profesores y estudiantes concluyen que se logró el objetivo de que los alumnos aplicaran el s2000 plus, cabe señalar que esto implica que no solo la aplicación de los instrumentos sino la forma en que se hace, es decir, respectando confidencialidad, asistiendo puntualmente a su práctica, el uso de vestimenta adecuada, evitar accesorios llamativos en el rostro, apagar su celular mientras evalúan, etc. Los estudiantes que consideran no se cumplió el objetivo puede deberse a una confusión ya que, si bien hubo casos en los 
que los evaluados no acudieron a su cita y tuvieron que ser reemplazados por supervisores para principios prácticos, todos los estudiantes aplicaron el s2000 plus aunque la percepción de ellos fue diferente a la de la mayoría. Dicha percepción puede tener origen en que, al realizar un juego de roles en el que el supervisor hacía las veces de evaluado, algunos de los estudiantes no consideraron dicha práctica como un objetivo cumplido pese a que la pregunta implicaba solo el hecho de aplicar el instrumento s2000 plus dentro del espacio, independientemente de quien haya sido la persona a la que se le aplicó dicho instrumento. El LOE al ser un escenario real se ve afectado ocasional o parcialmente por contratiempos igualmente reales que cualquier servicio o profesionista relacionado puede padecer: falta de recurso humano, económico o material, desastres naturales, falta de usuarios o el incumplimiento de los mismos a las citas pactadas, etc. Si bien algunos estudiantes pudieron estar inconformes con la manera en que se cumplió el objetivo la realidad es que tarde o temprano vivirán contratiempos similares en otras prácticas y es mejor que desde el inicio tomen conciencia de que no todo sale siempre tal cual es planeado y deben existir alternativas de solución, alternativas no creativas, sino unas adecuadas y efectivas de acuerdo a la situación específica para cumplir con el objetivo.

En cuanto a la correspondencia entre el material visto en Práctica Supervisada I con el trabajo realizado en el escenario, tanto profesores como estudiantes coinciden con que existe correspondencia, esto debido principalmente a que todo lo hecho o revisado en clase fue muy similar a lo que los estudiantes realizaron en el LOE. Es importante considerar que aunque solo $1.1 \%$ de los estudiantes considera que no existe dicha correspondencia, $8.7 \%$ de ellos asegura que las indicaciones que contiene el manual de entrenamiento del s2000 plus no corresponden completamente a las del manual. Esto puede indicar que las diferencias son tan infimas que en realidad no los afectó o a que dichas diferencias fueron superadas exitosamente en el LOE, ya que si bien existen algunas diferencias mínimas entre lo realizado en el espacio con lo que está escrito en el manual de entrenamiento del s2000 plus y las prácticas que los profesores realizaron en clase esto es debido a que los colaboradores del LOE cuentan con una amplia experiencia en aplicaciones grupales y el manejo del instrumento de manera cotidiana, la cual es necesaria para adecuarse al contexto en el que se aplicó la práctica (generalmente aplicaciones grupales siendo una numerosa mayoría de estudiantes y evaluados con respecto a los supervisores). El análisis de la utilidad del manual resulta fundamental pues es elaborado con fines didacticos por los responsables del laboratorio y en él no solo se incluye toda la información acerca de los instrumentos a aplicar, sino se integran apartados de ejeric- 
tación en aula de manera que permite que el alumno ensaye solo o con otros comapeñros dentro o fuera del aula.Así mismo se incluyen listas de chequeo para evaluar la adquisición de la competencia tratando de asegurar que cuando se presenten al escenario se puedan disminuir en lo posible errores en la aplicación proporcionando mayor certidumbre y apoyo a los alumnos. Para finalizar contiene la evidencia que les permite acreditar su práctica y da certeza del cumplimiento cabal de la misma. Es mportante que los espacios que fungan como escenarios de práctica sean evaluados por sus principales actores y para quienes se justifican: loa alumnos.

Los tres grupos (estudiantes, profesores y supervisores) coinciden en su totalidad con que el manual de entrenamiento del s2000 plus fue una herramienta útil para su aprendizaje de los estudiantes. En general lo que más destacan los tres grupos es la utilidad práctica del manual y la calidad y el detalle de la información que contiene. En esta ocasión también se presentó una aparente contrariedad en los datos proporcionados por los estudiantes ya que si bien la totalidad de ellos considera que el manual de entrenamiento del s2000 plus fue una herramienta útil para su aprendizaje, 8.2\% considera que algunas instrucciones en el escenario difieren de las del manual, esto puede justificarse de la misma forma que ya fue hecho con anterioridad con respecto a lo hecho en clase, lo escrito en el manual y lo hecho en el espacio.

Los aspectos interesantes o positivos destacados por estudiantes y profesores fueron muy diversos pero coincidieron en cuanto al tipo de instrumentos empleados en el espacio.

Las sugerencias generales para mejorías del espacio como escenario de prácticas de estudiantes, supervisores y estudiantes coincidieron en que lo mas necesario es una mayor cantidad de áreas dónde evaluar, esto es natural debido a que en el proceso de las prácticas es necesario que se encuentren presentes al mismo tiempo estudiantes, supervisores y evaluados.

Sería conveniente incluir en el manual de entrenamiento del s2000 plus mayores especificaciones sobre la conveniencia de realizar o no ciertas actividades específicas de acuerdo con la experiencia de los colaboradores del LOE así como especificar las consecuencias variables que implica trabajar con personas reales que pueden o no desear evaluarse en determinadas fechas o no cumplir con citas pactadas, entre otras situaciones. 
Un aspecto altamente interesante que no fue planificado al momento de iniciar el estudio es el hecho de que se presenten una gran cantidad de respuestas emocionales o afectivas. Generalmente los estudiantes se encuentran nerviosos debido a que es su primera práctica en un escenario real con evaluados reales y obsesionados con aprender cada palabra de las instrucciones del manual, también de manera general en el transcurso de su práctica y al terminarla se manifiestan aliviados. La tensión y emociones que manifiestan los estudiantes como el miedo, pena, nerviosismo, etc. los lleva a percibir de manera subjetiva todo lo que se realizó en el espacio así como a describir lo que sucedió en el espacio de una manera consistente con el desenlace emocional que tuvo al terminar la práctica..

En posteriores estudios es importante tomar en consideración los factores emocionales que manifiestan los estudiantes para analizarlos y complementar el proceso previo a la práctica ya sea con instrucciones, programas o técnicas para minimizar conflictos emocionales y discordancias entre estudiantes, profesores y supervisores.

En relación al instrumento empleado para la evaluación del cumplimiento de la práctica, a pesar de proporcionar información importante se acepta que es necesario hacer mejoras, especilmente relacionadas con hacer las repuestas de opción múltiple para dirigir o centrar la atención de estudiantes, pues esto puede ayudar a mejorar la confiabilidad del instrumento; afortunadamente esta experiencia permite tener una idea más precisa de los aspectos relevantes para los alumnos al ser su primer contacto con usuarios reales. Cabe señalar la revelancia de evaluar estos escenarios donde los alumnos se presentan a aplicar lo aprendido en aulas, pues se debe asegurar que en los escenarios las compencias son aplicadas y sensibiliar a los alumnos al trabajo real haciendo que esta transición sea gradual agradable y educativa. 


\section{Referencias}

Frías, M. y Jasa, G. (2005). Una evaluación preliminar de la implementación del plan de estudios de derecho basado en competencias. En E. A. Carlos., L. A. Galván, D. González., (Eds.), Investigaciones educativas en Sonora., Volumen VII.

Guevara, C. (2006). Análisis interconductual de algunos elementos que constituyen la enseñanza básica. Revista mexicana de investigación educativa, 20, 1037-1064

Ibáñez, C. (2007a). Metodología para la planeación de la educación superior. Una aproximación desde la psicología interconductual. México: Departamento de Psicología y Ciencias de la Comunicación, División de Ciencias Sociales, Universidad de Sonora

Ibáñez, C. (2007b). Un análisis crítico del modelo del triangulo pedagógico. Una propuesta alternativa. Revista mexicana de investigación educativa, 32, 435-456

Ibáñez, C. (2004) La planeación del currículo universitario basado en competencias conductuales. En Irigoyen, J. J. y Jiménez, M. Análisis funcional del comportamiento y educación. Hermosillo: Editorial UniSon

Macotela, S. (2007). Replanteando la formación de psicólogos: un análisis de problemas y algunas alternativas de solución. Enseñanza e investigación en psicología, 12, 5-25.

Macotela, S. y Paredes, H. (2003). Formación de psicólogos escolares con base en un modelo de supervisión experta en campo. Enseñanza e investigación en psicología, 8(1), 5-25.

Peralta, F. (2005). Hacia un desarrollo profesional convergente de la orientación escolar. Electronic Journal of Research in Educational Psychology, 3(1), 205-226.

Piña, J., A. y Rivera, B., M. (2006). El papel del análisis teórico-conceptual en el diseño de instrumentos en psicología y salud desde un modelo competencial. Revista colombiana de psicología, 15, 113-123

Plan de Estudios, Licenciatura en Psicología. (2004) Departamento de Psicología y Ciencias de la Comunicación, División de Ciencias Sociales, Universidad de Sonora (Unidad Regional Centro)

Programa de Práctica Supervisada I. (2007) Departamento de Psicología y Ciencias de la Comunicación, División de Ciencias Sociales, Universidad de Sonora (Unidad Regional Centro)

Ribes, E. y López, F. (1985) Teoría de la conducta. Un análisis de campo y paramétrico. México: Trillas 
Jesús Juan F. Arredondo Damián et al.

Sander, P. (2005). La investigación sobre nuestros alumnos, en pro de una mayor eficaciaa en la enseñanza universitaria. Electronic Journal of Research in Educational Psychology, 3(1), 113-130. 